\title{
A generalized flat extension theorem for moment matrices
}

\author{
Monique Laurent And Bernard Mourrain
}

\begin{abstract}
In this note we prove a generalization of the flat extension theorem of Curto and Fialkow (Memoirs of the American Mathematical Society, vol. 119. American Mathematical Society, Providence, 1996) for truncated moment matrices. It applies to moment matrices indexed by an arbitrary set of monomials and its border, assuming that this set is connected to 1 . When formulated in a basis-free setting, this gives an equivalent result for truncated Hankel operators.
\end{abstract}

Mathematics Subject Classification (2000). Primary 30E05; Secondary $12 \mathrm{D} 10$.

Keywords. Truncated moment problem, Moment matrix, Hankel operator, Polynomial optimization.

1. Introduction. Throughout this note, $\mathbb{K}$ denotes a field, $\mathbb{K}[\mathbf{x}]=\mathbb{K}\left[x_{1}, \ldots, x_{n}\right]$ is the ring of multivariate polynomials in $n$ variables $\mathbf{x}=\left(x_{1}, \ldots, x_{n}\right)$ with coefficients in $\mathbb{K}, \mathcal{M}_{n}=\left\{\mathbf{x}^{\alpha}:=x_{1}^{\alpha_{1}} \cdots x_{n}^{\alpha_{n}} \mid \alpha \in \mathbb{N}^{n}\right\}$ is the set of monomials in the variables $\mathbf{x}$, and $\mathcal{M}_{n, t}$ (resp., $\mathbb{K}[\mathbf{x}]_{t}$ ) is the set of monomials (resp., of polynomials) of degree at most $t$. The dual basis of $\mathcal{M}_{n}$ in the dual space $\mathbb{K}[\mathbf{x}]^{*}$ is denoted as $\mathcal{D}_{n}=\left\{\mathbf{d}^{\beta} \mid \beta \in \mathbb{N}^{n}\right\}$.

The natural action of $\mathbb{K}[\mathbf{x}]$ on $\mathbb{K}[\mathbf{x}]^{*}$ is denoted by

$$
(p, \Lambda) \in \mathbb{K}[\mathbf{x}] \times \mathbb{K}[\mathbf{x}]^{*} \mapsto p \cdot \Lambda \in \mathbb{K}[\mathbf{x}]^{*}
$$

where $(p \cdot \Lambda)(q):=\Lambda(p q)$ for $q \in \mathbb{K}[\mathbf{x}]$.

1.1. The moment problem. In this section, we consider $\mathbb{K}=\mathbb{R}$. The moment problem (see, e.g. [1,7]) deals with the characterization of the sequences of moments of measures. Given a probability measure $\mu$ on $\mathbb{R}^{n}$, its moment of order $a=\mathbf{x}^{\alpha} \in \mathcal{M}_{n}$ is the quantity $\int \mathbf{x}^{\alpha} \mu(d x)$. The moment problem concerns the characterization of the sequences $y=\left(y_{a}\right)_{a \in \mathcal{M}_{n}}$ that are the sequences of moments of some nonnegative Borel measure $\mu$, in which case one says that $\mu$ is a representing measure for $y$. Let $\Lambda \in \mathbb{R}[\mathbf{x}]^{*}$ denote the linear form on 
$\mathbb{R}[\mathbf{x}]$ associated to the sequence $y$, defined by $\Lambda(p)=\sum_{a} p_{a} y_{a}$ for any polynomial $p=\sum_{a \in \mathcal{M}_{n}} p_{a} a \in \mathbb{R}[\mathbf{x}]$. Then, $y$ has a representing measure $\mu$ precisely when $\Lambda$ is given by $\Lambda(p)=\int p(x) \mu(d x)$ for all $p \in \mathbb{R}[\mathbf{x}]$. A well known necessary condition for the existence of a representing measure is the positivity of $\Lambda$, i.e. $\Lambda\left(p^{2}\right) \geq 0$ for all $p \in \mathbb{R}[\mathbf{x}]$, which is equivalent to requiring that the (infinite) matrix $M(y):=\left(y_{a b}\right)_{a, b \in \mathcal{M}_{n}}$ be positive semidefinite. As is well known, this necessary condition is also sufficient in the univariate case $(n=1)$ (Hamburger's theorem [8]). However, it is not sufficient in the multivariate case, since for any $n \geq 2$ there exist nonnegative polynomials on $\mathbb{R}^{n}$ that are not sums of squares of polynomials (cf., e.g. [17] for details). However, positivity is sufficient for the existence of a representing measure under some additional assumptions. This is the case, for instance, when the sequence $y$ is (exponentially) bounded [2,3]. The next result of Curto and Fialkow [4] shows that this is also the case when the matrix $M(y)$ has finite rank (cf. also $[15,16]$ for a short proof).

Theorem 1.1. [4] If $M(y)$ is positive semidefinite and the rank of $M(y)$ is finite, then $y$ has a (unique) representing measure (which is finitely atomic with rank $M(y)$ atoms).

In the univariate case $n=1$, a matrix of the form $M(y)$ is a Hankel matrix. In the multivariate case, $M(y)$ is known as a generalized Hankel matrix (see [19]) or moment matrix (see [16]). One can also define truncated moment matrices: A matrix $M$ indexed by a subset $\mathcal{C} \subseteq \mathcal{M}_{n}$ is said to be a moment matrix if $M_{a, b}=M_{a^{\prime}, b^{\prime}}$ for all $a, b, a^{\prime}, b^{\prime} \in \mathcal{C}$ with $a b=a^{\prime} b^{\prime}$. Thus its entries are given by a sequence $y=\left(y_{c}\right)_{c \in \mathcal{C} \cdot \mathcal{C}}$, where $\mathcal{C} \cdot \mathcal{C}:=\{a b \mid a, b \in \mathcal{C}\}$, and we can write $M=M_{\mathcal{C}}(y)$. When $\mathcal{C}=\mathcal{M}_{n, t}$, we also write $M=M_{t}(y)$, where the entries of $y$ are indexed by $\mathcal{M}_{n, 2 t}$. Such matrices arise naturally in the context of the truncated moment problem, which asks for the existence of a representing measure for a truncated sequence indexed by a subset of monomials. A solution to the truncated moment problem would in fact imply a solution to the moment problem. Indeed, Stochel [20] shows that a sequence $y=\left(y_{a}\right)_{a \in \mathcal{M}_{n}}$ has a representing measure if and only if the truncated sequence $\left(y_{a}\right)_{a \in \mathcal{M}_{n, t}}$ has a representing measure for all $t \in \mathbb{N}$.

1.2. The flat extension theorem of Curto and Fialkow. Curto and Fialkow studied intensively the truncated moment problem (cf., e.g. [4-6] and further references therein). In particular, they observed that the notion of flat extension of matrices plays a central role in this problem. Given matrices $M_{\mathcal{C}}$ and $M_{\mathcal{B}}$ indexed, respectively, by $\mathcal{C}$ and $\mathcal{B} \subseteq \mathcal{C}, M_{\mathcal{C}}$ is said to be a flat extension of $M_{\mathcal{B}}$ if $M_{\mathcal{B}}$ coincides with the principal submatrix of $M_{\mathcal{C}}$ indexed by $\mathcal{B}$ and rank $M_{\mathcal{C}}=$ rank $M_{\mathcal{B}}$. Curto and Fialkow [4] show the following result for truncated moment matrices.

Theorem 1.2. (The flat extension theorem [4]) For a sequence $y=\left(y_{a}\right)_{a \in \mathcal{M}_{n, 2 t}}$, if $M_{t}(y)$ is a flat extension of $M_{t-1}(y)$, then there exists a (unique) sequence $\tilde{y}=\left(\tilde{y}_{a}\right)_{a \in \mathcal{M}_{n}}$ for which $M(\tilde{y})$ is a flat extension of $M_{t}(y)$. 
The flat extension theorem combined with Theorem 1.1 directly implies the following sufficient condition for existence of a representing measure.

Corollary 1.3. For a sequence $y=\left(y_{a}\right)_{a \in \mathcal{M}_{n, 2 t}}$, if $M_{t}(y)$ is positive semidefinite and $M_{t}(y)$ is a flat extension of $M_{t-1}(y)$, then $y$ has a representing measure.

Curto and Fialkow [5] show moreover that the flat extension condition is in some sense necessary and sufficient for the existence of a representing measure. More precisely, they show that a sequence $y=\left(y_{a}\right)_{a \in \mathcal{M}_{n, 2 t}}$ has a representing measure if and only if it can be extended to a sequence $y^{\prime}=\left(y_{a}^{\prime}\right)_{a \in \mathcal{M}_{n, 2 t+2 k+2}}$ (for some $k \geq 0$ ) for which $M_{t+k+1}\left(y^{\prime}\right)$ is a flat extension of $M_{t+k}\left(y^{\prime}\right)$.

The proof of Theorem 1.2 relies on a "truncated ideal like" property of the kernel of flat moment matrices (see (2.2) below). This permits to set up a linear system of equations in order to construct the flat extension $M_{t+1}(\tilde{y})$ of $M_{t}(y)$ (and then iteratively the infinite flat extension $M(\tilde{y})$ ). This system is largely overdetermined and the proof of existence of a solution involves technical details. See also [16] for an exposition of this proof. We propose in this note a simple alternative proof, which applies more generally to truncated moment matrices indexed by (suitable) general monomial sets (see Theorem 1.4).

1.3. A generalized flat extension theorem. We need some definitions to state our extension of Theorem 1.2. For $\mathcal{C} \subseteq \mathcal{M}_{n}$,

$$
\mathcal{C}^{+}:=\mathcal{C} \cup \bigcup_{i=1}^{n} x_{i} \mathcal{C}=\left\{m, x_{1} m, \ldots, x_{n} m \mid m \in \mathcal{C}\right\} \quad \text { and } \quad \partial \mathcal{C}:=\mathcal{C}^{+} \backslash \mathcal{C}
$$

are called, respectively, the closure and the border of $\mathcal{C}$. The set $\mathcal{C} \subseteq \mathcal{M}_{n}$ is said to be connected to 1 if $1 \in \mathcal{C}$ and every monomial $m \in \mathcal{C} \backslash\{1\}$ can be written as $m=x_{i_{1}} \cdots x_{i_{k}}$ with $x_{i_{1}}, x_{i_{1}} x_{i_{2}}, \ldots, x_{i_{1}} \cdots x_{i_{k}} \in \mathcal{C}$. For instance, $\mathcal{C}$ is connected to 1 if $\mathcal{C}$ is closed under taking divisors. For example, $\left\{1, x_{2}, x_{1} x_{2}\right\}$ is connected to 1 but $\left\{1, x_{1} x_{2}\right\}$ is not. We now state our main result.

Theorem 1.4. Consider a sequence $y=\left(y_{a}\right)_{a \in \mathcal{C}^{+} \cdot \mathcal{C}^{+}}$, where $\mathcal{C} \subseteq \mathcal{M}_{n}$ is finite and connected to 1 . If $M_{\mathcal{C}^{+}}(y)$ is a flat extension of $M_{\mathcal{C}}(y)$, then there exists a (unique) sequence $\tilde{y}=\left(\tilde{y}_{a}\right)_{a \in \mathcal{M}_{n}}$ for which $M(\tilde{y})$ is a flat extension of $M_{\mathcal{C}^{+}}(y)$.

The proof is delayed till Section 2. Note that Theorem 1.2 follows directly from Theorem 1.4 applied to the case $\mathcal{C}=\mathcal{M}_{n, t-1}$. Thus our result can be seen as a sparse version of Theorem 1.2, which applies to a more general monomial set $\mathcal{C}$, not necessarily the full set of monomials up to a given degree. We now give an example showing that the assumption that $\mathcal{C}$ is connected to 1 cannot be omitted.

Example. For $n=1$, consider the set $\mathcal{C}=\left\{1, x^{3}\right\}$, which is not connected to 1 , with $\partial \mathcal{C}=\left\{x, x^{4}\right\}$. Consider the sequence $y \in \mathbb{R}^{\mathcal{C}^{+} \cdot \mathcal{C}^{+}}$defined by $y_{1}=y_{x}=$ $y_{x^{2}}=1, y_{x^{3}}=y_{x^{4}}=y_{x^{5}}=a$ and $y_{x^{6}}=y_{x^{7}}=y_{x^{8}}=b$, where $a, b$ are scalars with $b \neq a^{2}$. Then, rank $M_{\mathcal{C}^{+}}(y)=\operatorname{rank} M_{\mathcal{C}}(y)=2$. If there is an extension $M(\tilde{y})$ of $M_{\mathcal{C}^{+}}(y)$, then its principal submatrix indexed by $\mathcal{C}^{+} \cup\left\{x^{2}\right\}$ has the form: 


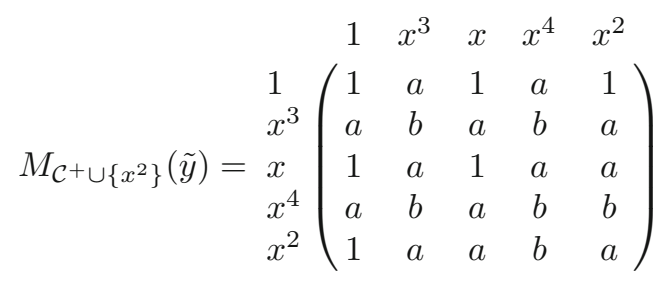

However, if it is a flat extension of $M_{\mathcal{C}^{+}}(\tilde{y})$, then the first and third columns coincide (giving $a=1$ ), as well as the second and fourth columns (giving $a=b$ ). Thus, $1=a=b$, contradicting our choice $b \neq a^{2}$. Hence no flat extension exists.

1.4. Basis-free reformulation. Here we reformulate our result in a basis-free setting. Moment matrices correspond indeed to choosing the monomial basis $\mathcal{M}_{n}$ in the polynomial ring $\mathbb{K}[\mathbf{x}]$ and its dual basis $\mathcal{D}_{n}$ in the dual space $\mathbb{K}[\mathbf{x}]^{*}$. Given $\Lambda \in \mathbb{K}[\mathbf{x}]^{*}$, the operator

$$
\begin{aligned}
H_{\Lambda}: \mathbb{K}[\mathbf{x}] & \rightarrow \mathbb{K}[\mathbf{x}]^{*} \\
p & \mapsto p \cdot \Lambda
\end{aligned}
$$

is known as a Hankel operator. Its matrix with respect to the bases $\mathcal{M}_{n}$ and $\mathcal{D}_{n}$ is precisely the moment matrix $\left(\Lambda\left(x^{\alpha+\beta}\right)\right)_{\alpha, \beta \in \mathcal{M}_{n}}=M(y)$ of the sequence $y=(\Lambda(a))_{a \in \mathcal{M}_{n}}$. The kernel of $H_{\Lambda}$,

$$
\text { ker } H_{\Lambda}=\{p \in \mathbb{K}[\mathbf{x}] \mid \Lambda(p q)=0 \forall q \in \mathbb{K}[\mathbf{x}]\} \text {, }
$$

is an ideal in $\mathbb{K}[\mathbf{x}]$. Moreover, when $\mathbb{K}=\mathbb{R}$ and $\Lambda$ is positive, i.e. when $\Lambda\left(p^{2}\right) \geq 0$ for all $p \in \mathbb{R}[\mathbf{x}]$, ker $H_{\Lambda}$ is a real radical ideal [15]. Theorem 1.1 means that $\Lambda \in \mathbb{R}[\mathbf{x}]^{*}$ is positive with rank $H_{\Lambda}<\infty$ if and only if there exists a nonnegative finite atomic measure $\mu$ for which $\Lambda(p)=\int p(x) \mu(d x)$ for all $p \in \mathbb{R}[\mathbf{x}]$.

Truncated Hankel operators can be analogously defined. Given $\mathcal{C} \subseteq \mathcal{M}_{n}$ and $\Lambda \in\left(\operatorname{Span}\left(\mathcal{C}^{+} \cdot \mathcal{C}^{+}\right)\right)^{*}$, the corresponding Hankel operator is

$$
\begin{aligned}
H_{\Lambda}^{\mathcal{C}^{+}}: \operatorname{Span}\left(\mathcal{C}^{+}\right) & \rightarrow \operatorname{Span}\left(\mathcal{C}^{+}\right)^{*} \\
p & \mapsto p \cdot \Lambda
\end{aligned}
$$

and its restriction to $\operatorname{Span}(\mathcal{C})$ is $H_{\Lambda}^{\mathcal{C}}: \operatorname{Span}(\mathcal{C}) \rightarrow \operatorname{Span}(\mathcal{C})^{*}$. We have the following mappings:

$$
\frac{\operatorname{Span}(\mathcal{C})}{\operatorname{ker} H_{\Lambda}^{\mathcal{C}}} \stackrel{\sigma_{1}}{\longleftarrow} \frac{\operatorname{Span}(\mathcal{C})}{\operatorname{ker} H_{\Lambda}^{\mathcal{C}^{+}} \cap \operatorname{Span}(\mathcal{C})} \stackrel{\sigma_{2}}{\longrightarrow} \frac{\operatorname{Span}\left(\mathcal{C}^{+}\right)}{\operatorname{ker} H_{\Lambda}^{\mathcal{C}^{+}}}
$$

where $\sigma_{1}$ is onto and $\sigma_{2}$ is one-to-one, so that

$$
\operatorname{dim} \frac{\operatorname{Span}(\mathcal{C})}{\operatorname{ker} H_{\Lambda}^{\mathcal{C}}} \leq \operatorname{dim} \frac{\operatorname{Span}(\mathcal{C})}{\operatorname{ker} H_{\Lambda}^{\mathcal{C}^{+}} \cap \operatorname{Span}(\mathcal{C})} \leq \operatorname{dim} \frac{\operatorname{Span}\left(\mathcal{C}^{+}\right)}{\operatorname{ker} H_{\Lambda}^{\mathcal{C}^{+}}} .
$$

Thus, rank $H_{\Lambda}^{\mathcal{C}^{+}}=\operatorname{rank} H_{\Lambda}^{\mathcal{C}}$ (in which case we also say that $H_{\Lambda}^{\mathcal{C}^{+}}$is a flat extension of $H_{\Lambda}^{\mathcal{C}}$ ) if and only if equality holds throughout in (1.2), i.e. both $\sigma_{1}$ and $\sigma_{2}$ in (1.1) are isomorphisms or, equivalently, if

$$
\operatorname{Span}\left(\mathcal{C}^{+}\right)=\operatorname{Span}(\mathcal{C})+\operatorname{ker} H_{\Lambda}^{\mathcal{C}^{+}} \text {and } \operatorname{ker} H_{\Lambda}^{\mathcal{C}}=\operatorname{ker} H_{\Lambda}^{\mathcal{C}^{+}} \cap \operatorname{Span}(\mathcal{C}) .
$$

Theorem 1.4 can be reformulated as follows. 
Theorem 1.5. Let $\Lambda \in\left(\operatorname{Span}\left(\mathcal{C}^{+} \cdot \mathcal{C}^{+}\right)\right)^{*}$, where $\mathcal{C} \subseteq \mathcal{M}_{n}$ is finite and connected to 1 , and assume that rank $H_{\Lambda}^{\mathcal{C}^{+}}=$rank $H_{\Lambda}^{\mathcal{C}}$. Then there exists $(a$ unique) $\tilde{\Lambda} \in \mathbb{R}[\mathbf{x}]^{*}$ for which $H_{\tilde{\Lambda}}$ is a flat extension of $H_{\Lambda}^{\mathcal{C}^{+}}$, i.e. $\tilde{\Lambda}$ coincides with $\Lambda$ on $\operatorname{Span}\left(\mathcal{C}^{+} \cdot \mathcal{C}^{+}\right)$and $\operatorname{rank} H_{\tilde{\Lambda}}=\operatorname{rank} H_{\Lambda}^{\mathcal{C}^{+}}$.

1.5. Border bases and commuting multiplication operators. We recall here a result of [18] about border bases of polynomial ideals that we exploit to prove our flat extension theorem. Let $\mathcal{B}:=\left\{b_{1}, \ldots, b_{N}\right\}$ be a finite set of distinct monomials. Assume that, for each border monomial $x_{i} b_{j} \in \partial \mathcal{B}$, we are given a polynomial of the form

$$
g^{(i j)}:=x_{i} b_{j}-\sum_{h=1}^{N} a_{h}^{(i j)} b_{h} \quad \text { where } a_{h}^{(i j)} \in \mathbb{K} .
$$

The set

$$
F:=\left\{g^{(i j)} \mid i=1, \ldots, n, j=1, \ldots, N \text { with } x_{i} b_{j} \in \partial \mathcal{B}\right\}
$$

is known as a border prebasis [10] or a rewriting family for $\mathcal{B}$ [18]. When the set $\mathcal{B}$ contains the constant monomial 1 , one can easily verify that $\mathcal{B}$ is a generating set for the quotient space $\mathbb{K}[\mathbf{x}] /(F)$, where $(F)$ is the ideal generated by the set $F$. When $\mathcal{B}$ is connected to 1 , Theorem 1.6 below characterizes the case when $\mathcal{B}$ is a basis of $\mathbb{K}[\mathbf{x}] /(F)$, in which case $F$ is said to be a border basis of the ideal $(F)$. For this, for each $i=1, \ldots, n$, consider the linear operator:

$$
\begin{aligned}
\chi_{i}: \operatorname{Span}(\mathcal{B}) & \rightarrow \operatorname{Span}(\mathcal{B}) \\
b_{j} & \mapsto \chi_{i}\left(b_{j}\right)= \begin{cases}x_{i} b_{j} & \text { if } x_{i} b_{j} \in \mathcal{B}, \\
\sum_{h=1}^{N} a_{h}^{(i j)} b_{h} & \text { if } x_{i} b_{j} \in \partial \mathcal{B}\end{cases}
\end{aligned}
$$

extended to $\operatorname{Span}(\mathcal{B})$ by linearity. When $\mathcal{B}$ is a basis of $\mathbb{K}[\mathbf{x}] /(F), \chi_{i}$ corresponds to the "multiplication operator by $x_{i}$ " from $\mathbb{K}[\mathbf{x}] /(F)$ to $\mathbb{K}[\mathbf{x}] /(F)$ and thus the operators $\chi_{1}, \ldots, \chi_{n}$ commute pairwise. The next result of $[18]$ shows that the converse implication holds when $\mathcal{B}$ is connected to 1 ; this was also proved later in [10] when $\mathcal{B}$ is closed under taking divisors.

Theorem 1.6. [18] Let $\mathcal{B} \subseteq \mathcal{M}_{n}$ be a finite set of monomials which is connected to 1 , let $F$ be a rewriting family for $\mathcal{B}$ as in $(1.3)$, and let $\chi_{1}, \ldots, \chi_{n}$ be defined as in (1.4). The set $\mathcal{B}$ is a basis of the quotient space $\mathbb{K}[\mathbf{x}] /(F)$ if and only if the operators $\chi_{1}, \ldots, \chi_{n}$ commute pairwise.

The proof of our generalized flat extension theorem is an adaptation of this result to kernels of Hankel operators, where we omit the assumption that $\mathcal{B}$ is connected to 1.

1.6. Contents of the paper. Section 2 contains the proof of our generalized flat extension theorem and we mention some applications in Sect. 3. In particular, we observe that Theorem 1.2 is an 'easy' instance of our flat extension theorem (since one can prove existence of a basis connected to 1). We also point out the relevance of the flat extension theorem to polynomial optimization and to the problem of computing real roots to systems of polynomial equations. 
2. Proof of the flat extension theorem. We give here the proof of Theorem 1.5 (equivalently, of Theorem 1.4). We will often use the following simple observations, which follow directly from the assumption that rank $H_{\Lambda}^{\mathcal{C}^{+}}=\operatorname{rank} H_{\Lambda}^{\mathcal{C}}$ : For all $p \in \operatorname{Span}\left(\mathcal{C}^{+}\right)$,

$$
\begin{aligned}
& p \in \operatorname{ker} H_{\Lambda}^{\mathcal{C}^{+}} \stackrel{\text { def. }}{\Longleftrightarrow} \Lambda(a p)=0 \forall a \in \mathcal{C}^{+} \Longleftrightarrow \Lambda(a p)=0 \quad \forall a \in \mathcal{C}, \\
& p \in \operatorname{ker} H_{\Lambda}^{\mathcal{C}^{+}} \quad \text { and } \quad x_{i} p \in \operatorname{Span}\left(\mathcal{C}^{+}\right) \Longrightarrow x_{i} p \in \operatorname{ker} H_{\Lambda}^{\mathcal{C}^{+}} .
\end{aligned}
$$

Our objective is to construct a linear form $\tilde{\Lambda} \in \mathbb{K}[\mathbf{x}]^{*}$ whose Hankel operator $H_{\tilde{\Lambda}}$ is a flat extension of $H_{\Lambda}^{\mathcal{C}^{+}}$.

Let $\mathcal{B} \subseteq \mathcal{C}$ for which $\operatorname{rank} H_{\Lambda}^{\mathcal{C}^{+}}=\operatorname{rank} H_{\Lambda}^{\mathcal{B}}=|\mathcal{B}|$. Note that we can assume that $1 \in \mathcal{B}$. Indeed, if no such $\mathcal{B}$ exists containing 1 , then $\Lambda(p)=0$ $\forall p \in \operatorname{Span}\left(\mathcal{C}^{+}\right)$and one can easily verify that this implies that $\Lambda$ is identically zero, in which case the theorem trivially holds.

From the assumption: $\operatorname{rank} H_{\Lambda}^{\mathcal{C}^{+}}=\operatorname{rank} H_{\Lambda}^{\mathcal{B}}=|\mathcal{B}|$, we have the direct sum decomposition: $\operatorname{Span}\left(\mathcal{C}^{+}\right)=\operatorname{Span}(\mathcal{B}) \oplus \operatorname{ker} H_{\Lambda}^{\mathcal{C}^{+}}$, and thus

$$
\begin{aligned}
& \forall p \in \operatorname{Span}\left(\mathcal{C}^{+}\right) \quad \exists ! \pi(p) \in \operatorname{Span}(\mathcal{B}) \text { such that } \\
& f(p):=p-\pi(p) \in \operatorname{ker} H_{\Lambda}^{\mathcal{C}^{+}} .
\end{aligned}
$$

Then the set

$$
F:=\{f(m)=m-\pi(m) \mid m \in \partial \mathcal{B}\}
$$

is a rewriting family for $\mathcal{B}$ and, for $i=1, \ldots, n$, the linear operator $\chi_{i}$ in (1.4) maps $p \in \operatorname{Span}(\mathcal{B})$ to $\chi_{i}(p)=\pi\left(x_{i} p\right) \in \operatorname{Span}(\mathcal{B})$. We show that $\chi_{1}, \ldots, \chi_{n}$ commute pairwise. Set $K:=\operatorname{ker} H_{\Lambda}^{\mathcal{C}^{+}}$.

Lemma 2.1. $\chi_{i} \circ \chi_{j}=\chi_{j} \circ \chi_{i}$.

Proof. Let $m \in \mathcal{B}$. Write $\pi\left(x_{i} m\right):=\sum_{b \in \mathcal{B}} \lambda_{b}^{i} b\left(\lambda_{b}^{i} \in \mathbb{R}\right)$. We have:

$$
\begin{aligned}
\chi_{j} \circ \chi_{i}(m) & =\chi_{j}\left(\sum_{b \in \mathcal{B}} \lambda_{b}^{i} b\right)=\sum_{b \in \mathcal{B}} \lambda_{b}^{i} \chi_{j}(b)=\sum_{b \in \mathcal{B}} \lambda_{b}^{i}\left(x_{j} b-f\left(x_{j} b\right)\right) \\
& =x_{j}\left(\sum_{b \in \mathcal{B}} \lambda_{b}^{i} b\right)-\sum_{b \in \mathcal{B}} \lambda_{b}^{i} f\left(x_{j} b\right) \\
& =x_{j}\left(x_{i} m-f\left(x_{i} m\right)\right)-\sum_{b \in \mathcal{B}} \lambda_{b}^{i} f\left(x_{j} b\right) .
\end{aligned}
$$

Therefore,

$$
\begin{aligned}
p:= & \chi_{j} \circ \chi_{i}(m)-\chi_{i} \circ \chi_{j}(m)=\underbrace{x_{i} f\left(x_{j} m\right)-x_{j} f\left(x_{i} m\right)}_{p_{1}} \\
& +\underbrace{\sum_{b \in \mathcal{B}} \lambda_{b}^{j} f\left(x_{i} b\right)-\lambda_{b}^{i} f\left(x_{j} b\right)}_{p_{2}} .
\end{aligned}
$$


We show that $p_{1} \in K$. Indeed, $\forall a \in \mathcal{C}, \Lambda\left(a p_{1}\right)=\Lambda\left(a x_{i} f\left(x_{j} m\right)-a x_{j} f\left(x_{i} m\right)\right)=0$ since $a x_{i}, a x_{j} \in \mathcal{C}^{+}$and $f\left(x_{i} m\right), f\left(x_{j} m\right) \in K$; by (2.1), this shows that $p_{1} \in K$. As $p_{2} \in K$ too, this implies $p \in K$ and thus $p=0$, because $p \in \operatorname{Span}(\mathcal{B})$.

Our objective now is to show that $\mathcal{B}$ is a basis of $\mathbb{K}[\mathbf{x}] /(F)$ and that, if $\tilde{\pi}$ denotes the projection from $\mathbb{K}[\mathbf{x}]$ onto $\operatorname{Span}(\mathcal{B})$ along $(F)$, then the operator $\tilde{\Lambda}$ defined by $\tilde{\Lambda}(p)=\Lambda(\tilde{\pi}(p))$ for $p \in \mathbb{K}[\mathbf{x}]$, defines the desired flat extension of $\Lambda$. Note that when $\mathcal{B}$ is connected to 1 , Theorem 1.6 implies directly that $\mathcal{B}$ is a basis of $\mathbb{K}[\mathbf{x}] /(F)$. As we do not assume $\mathcal{B}$ connected to 1 , we cannot apply Theorem 1.6, but our arguments below are inspired from its proof. In particular, we construct the projection $\tilde{\pi}$ via the mapping $\varphi$ from (2.4) below.

As the $\chi_{i}$ 's commute, the operator $f(\chi):=f\left(\chi_{1}, \ldots, \chi_{n}\right)$ is well defined for any polynomial $f \in \mathbb{K}[\mathbf{x}]$. Then $\mathbb{K}[\mathbf{x}]$ acts on $\operatorname{Span}(\mathcal{B})$ by

$$
(f, p) \in \mathbb{K}[\mathbf{x}] \times \operatorname{Span}(\mathcal{B}) \mapsto f(\chi)(p) \in \operatorname{Span}(\mathcal{B}) .
$$

Recall that $1 \in B$. The mapping

$$
\begin{aligned}
\varphi: \mathbb{K}[\mathbf{x}] & \rightarrow \operatorname{Span}(\mathcal{B}) \\
f & \mapsto f(\chi)(1)
\end{aligned}
$$

is a homomorphism and, by the following property,

$$
\varphi(f g)=f(\chi)(g(\chi)(1))=f(\chi)(\varphi(g)) \quad \forall f, g \in \mathbb{K}[\mathbf{x}],
$$

$\operatorname{ker} \varphi$ is an ideal in $\mathbb{K}[\mathbf{x}]$. We now prove that $\varphi$ coincide on $\operatorname{Span}\left(\mathcal{C}^{+}\right)$with the projection $\pi$ on $\operatorname{Span}(\mathcal{B})$ along $K=\operatorname{ker} H_{\Lambda}^{\mathcal{C}^{+}}$.

Lemma 2.2. For any element $m \in \mathcal{C}^{+}, \varphi(m)=\pi(m)$.

Proof. We use induction on the degree of $m$. If $m=1$, we have $\varphi(1)=\pi(1)=1$ since $1 \in \mathcal{B}$. Let $m \neq 1 \in \mathcal{C}^{+}$. As $\mathcal{C}$ is connected to $1, m$ is of the form $m=x_{i} m_{1}$ for some $m_{1} \in \mathcal{C}^{+}$. By the induction assumption, we have $\varphi\left(m_{1}\right)=\pi\left(m_{1}\right)$. Then,

$$
\varphi(m)=\varphi\left(x_{i} m_{1}\right)=\chi_{i}\left(\varphi\left(m_{1}\right)\right)=\chi_{i}\left(\pi\left(m_{1}\right)\right)=x_{i} \pi\left(m_{1}\right)-\kappa,
$$

with $\kappa \in F \subseteq K$. But we also have

$$
m=x_{i} m_{1}=x_{i}\left(\pi\left(m_{1}\right)+m_{1}-\pi\left(m_{1}\right)\right)=x_{i} \pi\left(m_{1}\right)+x_{i} \kappa_{1}
$$

where $\kappa_{1}:=m_{1}-\pi\left(m_{1}\right) \in K \cap \operatorname{Span}(\mathcal{C})$. We deduce that

$$
m=\varphi(m)+\kappa+x_{i} \kappa_{1}=\varphi(m)+\kappa_{2}
$$

with $\kappa_{2}:=\kappa+x_{i} \kappa_{1}$. As $\kappa_{1} \in K$ and $x_{i} \kappa_{1} \in \operatorname{Span}\left(\mathcal{C}^{+}\right)$, we deduce using (2.2) that $x_{i} \kappa_{1} \in K$. As $\kappa \in K$, this implies $\kappa_{2} \in K$. Finally, as $\varphi(m) \in \operatorname{Span}(\mathcal{B})$, it coincides with the projection $\pi(m)$ of $m$ on $\operatorname{Span}(\mathcal{B})$ along $K$.

This implies directly:

$$
\begin{aligned}
\varphi(b) & =b, \quad \varphi\left(x_{i} b\right)=\chi_{i}(b) \quad \forall b \in \mathcal{B} \quad \forall i=1, \ldots, n \\
\Lambda(p q) & =\Lambda(p \varphi(q))=\Lambda(\varphi(p) \varphi(q)) \quad \forall p, q \in \operatorname{Span}\left(\mathcal{C}^{+}\right) .
\end{aligned}
$$

Lemma 2.3. For all $p, q \in \operatorname{Span}\left(\mathcal{C}^{+}\right), \Lambda(p q)=\Lambda(\varphi(p q))$. 
Proof. We first show by induction on the degree of $m \in \mathcal{C}^{+}$that

$$
\Lambda(m b)=\Lambda(\varphi(m b)) \quad \forall b \in \mathcal{B} .
$$

The result is obvious if $m=1$. Else, as $\mathcal{C}^{+}$is connected to 1 , we can write $m=x_{i} m_{1}$ where $m_{1} \in \mathcal{C}^{+}$. Using first (2.7) and then (2.6), we find:

$$
\Lambda(m b)=\Lambda\left(m_{1} x_{i} b\right)=\Lambda\left(m_{1} \varphi\left(x_{i} b\right)\right)=\Lambda\left(m_{1} \chi_{i}(b)\right) .
$$

Next, using first the induction assumption and then (2.5), (2.6), we find:

$\Lambda\left(m_{1} \chi_{i}(b)\right)=\Lambda\left(\varphi\left(m_{1} \chi_{i}(b)\right)\right)=\Lambda\left(m_{1}(\chi)\left(\chi_{i}(b)\right)\right)=\Lambda(m(\chi)(b))=\Lambda(\varphi(m b))$

thus showing (2.8). We can now conclude the proof of the lemma. Let $p, q \in$ $\operatorname{Span}\left(\mathcal{C}^{+}\right)$. Then, using successively $(2.7),(2.8),(2.5),(2.6), \Lambda(p q)$ is equal to

$$
\Lambda(p \varphi(q))=\Lambda(\varphi(p \varphi(q)))=\Lambda(p(\chi)(\varphi(\varphi(q))))=\Lambda(p(\chi)(\varphi(q)))=\Lambda(\varphi(p q)) .
$$

We can now conclude the proof of Theorem 1.5. Let $\tilde{\Lambda}$ be the linear operator on $\mathbb{K}[\mathbf{x}]$ defined by

$$
\tilde{\Lambda}(p):=\Lambda(\varphi(p)) \quad \text { for } p \in \mathbb{K}[\mathbf{x}]
$$

We show that $H_{\tilde{\Lambda}}$ is the unique flat extension of $H_{\Lambda}^{\mathcal{C}^{+}}$.

First, $H_{\tilde{\Lambda}}$ is an extension of $H_{\Lambda}^{\mathcal{C}^{+}}$since, for all $p, q \in \operatorname{Span}\left(\mathcal{C}^{+}\right), \tilde{\Lambda}(p q)=$ $\Lambda(\varphi(p q))=\Lambda(p q)$ (by Lemma 2.3).

Next, we have $K=\operatorname{ker} H_{\Lambda}^{\mathcal{C}^{+}} \subseteq \operatorname{ker} H_{\tilde{\Lambda}}$. Indeed, let $\kappa \in K$. By Lemma 2.2, $\varphi(\kappa)=\pi(\kappa)=0$. Thus for any $p \in \mathbb{K}[\mathbf{x}]$, we have $\tilde{\Lambda}(p \kappa)=\Lambda(\varphi(p \kappa))=$ $\Lambda(p(\chi)(\varphi(\kappa)))=0$, which shows that $\kappa \in \operatorname{ker} H_{\tilde{\Lambda}}$.

As $F$ is a rewriting family for $\mathcal{B}$ and $\mathcal{B}$ contains $1, \mathcal{B}$ is a generating set of $\mathbb{K}[\mathbf{x}] /(F)$ and thus $\operatorname{dim} \mathbb{K}[\mathbf{x}] /(F) \leq|\mathcal{B}|$. Set $\mathcal{A}_{\tilde{\Lambda}}:=\mathbb{K}[\mathbf{x}] / \operatorname{ker} H_{\tilde{\Lambda}}$. Then, as $F \subseteq K \subseteq \operatorname{ker} H_{\tilde{\Lambda}}$, we have $\operatorname{dim} \mathcal{A}_{\tilde{\Lambda}} \leq \operatorname{dim} \mathbb{K}[\mathbf{x}] /(F) \leq|\mathcal{B}|$. On the other hand, $\operatorname{dim} \mathcal{A}_{\tilde{\Lambda}}=\operatorname{rank} H_{\tilde{\Lambda}} \geq \operatorname{rank} H_{\tilde{\Lambda}}^{\mathcal{B}}=\operatorname{rank} H_{\Lambda}^{\mathcal{B}}=|\mathcal{B}|$. Therefore, $\operatorname{dim} \mathcal{A}_{\tilde{\Lambda}}=$ $\operatorname{rank} H_{\tilde{\Lambda}}=|\mathcal{B}|, \operatorname{ker} H_{\tilde{\Lambda}}=(K), H_{\tilde{\Lambda}}$ is a flat extension of $H_{\Lambda}^{\mathcal{C}^{+}}$, and we have the direct sum: $\mathbb{K}[\mathbf{x}]=\operatorname{Span}(\mathcal{B}) \oplus \operatorname{ker} H_{\tilde{\Lambda}}$. Moreover, $\varphi(p)$ is the projection of $p \in$ $\mathbb{K}[\mathbf{x}]$ on $\operatorname{Span}(\mathcal{B})$ along $\operatorname{ker} H_{\tilde{\Lambda}}$. Indeed, $\varphi(p) \in \operatorname{Span}(\mathcal{B})$ and $p-\varphi(p) \in \operatorname{ker} H_{\tilde{\Lambda}}$ for any $p \in \mathbb{K}[\mathbf{x}]$ since, for any $q \in \mathbb{K}[\mathbf{x}]$,

$$
\begin{aligned}
\tilde{\Lambda}(p q) & =\Lambda(\varphi(p q))=\Lambda(p(\chi)(\varphi(q))), \\
\tilde{\Lambda}(p \varphi(q)) & =\Lambda(\varphi(p \varphi(q)))=\Lambda(p(\chi)(\varphi(q)))=\tilde{\Lambda}(p q) .
\end{aligned}
$$

Finally, if $\Lambda^{\prime} \in \mathbb{K}[\mathbf{x}]^{*}$ is another linear form whose Hankel operator $H_{\Lambda^{\prime}}$ is a flat extension of $H_{\Lambda}^{\mathcal{C}^{+}}$, then $\operatorname{ker} H_{\tilde{\Lambda}}=(K) \subseteq$ ker $H_{\Lambda^{\prime}}$. This implies that for all $p \in \mathbb{K}[\mathbf{x}], \Lambda^{\prime}(p)=\Lambda^{\prime}(\varphi(p))=\Lambda(\varphi(p))=\tilde{\Lambda}(p)$. This shows the uniqueness of the flat extension of $H_{\Lambda}^{\mathcal{C}^{+}}$, which concludes the proof of Theorem 1.5. 


\section{Applications.}

3.1. Application to the flat extension theorem of Curto and Fialkow. Theorem 1.2 is in some sense an 'easy' instance of Theorem 1.4. Indeed, under its assumptions, one can show existence of a maximum rank principal submatrix of $M_{t-1}(y)$ indexed by a monomial set $\mathcal{B}$ connected to 1 which, as noted in the proof of Theorem 1.4, permits to apply Theorem 1.6.

Proposition 3.1. Let $\Lambda \in\left(\operatorname{Span}\left(\mathcal{C}^{+} \cdot \mathcal{C}^{+}\right)\right)^{*}$, where $\mathcal{C}:=\mathcal{M}_{n, t-1}$. If $\operatorname{rank} H_{\Lambda}^{\mathcal{C}^{+}}=$ rank $H_{\Lambda}^{\mathcal{C}}$, then there exists $\mathcal{B} \subseteq \mathcal{C}$ closed under taking divisors (and thus connected to 1) for which $\operatorname{rank} H_{\Lambda}^{\mathcal{C}^{+}}=\operatorname{rank} H_{\Lambda}^{\mathcal{B}}=|\mathcal{B}|$.

Proof. Let $M=(\Lambda(a b))_{a, b \in \mathcal{C}^{+}}$denote the matrix of $H_{\Lambda}^{\mathcal{C}^{+}}$in the canonical bases. Consider a total degree monomial ordering $\preceq$ of $\mathcal{C}$ and let $\mathcal{B} \subseteq \mathcal{C}$ index a maximum linearly independent set of columns of $M$ which is constructed by the greedy algorithm using the ordering $\preceq$. One can easily verify that $\mathcal{B}$ is closed under taking divisors (cf. [14]).

The following example shows that, even if $\mathcal{C}$ is connected to 1 , there may not always exist a base $\mathcal{B}$ connected to 1 for $H_{\Lambda}^{\mathcal{C}}$ (which justifies our generalisation of Theorem 1.6 to kernels of Hankel operators).

Example. For $n=2$, let $\mathcal{C}=\left\{1, x_{1}, x_{1} x_{2}\right\}$ with $\partial \mathcal{C}=\left\{x_{2}, x_{1} x_{2}^{2}, x_{1}^{2}, x_{1}^{2} x_{2}\right\}$, and let $\Lambda \in\left(\operatorname{Span}\left(\mathcal{C}^{+} \cdot \mathcal{C}^{+}\right)\right)^{*}$ be defined by $\Lambda\left(x_{1}^{i} x_{2}^{j}\right)=1$ if $j=0,1$, and $\Lambda\left(x_{1}^{i} x_{2}^{j}\right)=a$ if $j=2,3,4$, except $\Lambda\left(x_{1}^{2} x_{2}^{4}\right)=a^{2}$, where $a$ is a scalar with $a \neq 1$. The associated moment matrix has the form

\begin{tabular}{|c|c|c|c|c|c|c|c|}
\hline & 1 & $x_{1}$ & $x_{1} x_{2}$ & $x_{1}^{2}$ & $x_{1}^{2} x_{2}$ & $x_{2}$ & $x_{1} x_{2}^{2}$ \\
\hline 1 & 1 & 1 & 1 & 1 & 1 & 1 & $a$ \\
\hline$x_{1}$ & 1 & 1 & 1 & 1 & 1 & 1 & $a$ \\
\hline$x_{1} x_{2}$ & 1 & 1 & $a$ & 1 & $a$ & $a$ & $a$ \\
\hline$x_{1}^{2}$ & 1 & 1 & 1 & 1 & 1 & 1 & $a$ \\
\hline$x_{1}^{2} x_{2}$ & 1 & 1 & $a$ & 1 & $a$ & $a$ & $a$ \\
\hline$x_{2}$ & 1 & 1 & $a$ & 1 & $a$ & $a$ & $a$ \\
\hline$x_{1} x_{2}^{2}$ & $a$ & $a$ & $a$ & $a$ & $a$ & $a$ & $a^{2}$ \\
\hline
\end{tabular}

and $\operatorname{rank} H_{\Lambda}^{\mathcal{C}}=\operatorname{rank} H_{\Lambda}^{\mathcal{C}^{+}}=2$. As $1-x_{1} \in \operatorname{ker} H_{\Lambda}^{\mathcal{C}^{+}}$, the only sets indexing a column base for $H_{\Lambda}^{\mathcal{C}}$ are $\mathcal{B}=\left\{1, x_{1} x_{2}\right\}$ and $\left\{x_{1}, x_{1} x_{2}\right\}$, thus not connected to 1 .

Combining Theorem 1.5 with Theorem 1.1 we obtain the following extension of Corollary 1.3.

Theorem 3.2. Let $\Lambda \in\left(\operatorname{Span}\left(\mathcal{C}^{+} \cdot \mathcal{C}^{+}\right)\right)^{*}$, where $\mathcal{C} \subseteq \mathcal{M}_{n}$ is finite and connected to 1. Assume that $\Lambda$ is positive and that rank $H_{\Lambda}^{\mathcal{C}^{+}}=\operatorname{rank} H_{\Lambda}^{\mathcal{C}}$. Then the sequence $y=(\Lambda(a))_{a \in \mathcal{C}^{+} \cdot \mathcal{C}^{+}}$has a representing measure.

3.2. Application to polynomial optimization. We point out here the relevance of the flat extension theorems to polynomial optimization and to the problem of computing the real roots to polynomial equations. In this section, we take again $\mathbb{K}=\mathbb{R}$. 
The truncated moment problem has recently attracted a lot of attention also within the optimization community, since it can be used to formulate semidefinite programming relaxations to polynomial optimization problems (see [12]). Moreover the flat extension theorem of Curto and Fialkow permits to detect optimality of the relaxations and to extract global optimizers to the original optimization problem (see [9]). Here is a brief sketch; see, e.g. [16] and references therein for details.

Suppose we want to compute the infimum $p^{*}$ of a polynomial $p$ over a semi-algebraic set $K$ defined by the polynomial inequalities $g_{1} \geq 0, \ldots, g_{m} \geq 0$. For any integer $t \geq \operatorname{deg}(p) / 2$ and such that $t \geq d_{j}:=\left\lceil\operatorname{deg}\left(g_{j}\right) / 2\right\rceil$, consider the program:

$$
p_{t}^{*}:=\inf \Lambda(p) \text { s.t. } \Lambda \in\left(\mathbb{R}[\mathbf{x}]_{2 t}\right)^{*}, \Lambda(1)=1, \Lambda \succeq 0, g_{j} \cdot \Lambda \succeq 0(\forall j \leq m) .
$$

Here, $\Lambda \succeq 0$ means that $\Lambda$ is positive (i.e., $\Lambda\left(p^{2}\right) \geq 0$ for all $\left.p \in \mathbb{R}[\mathbf{x}]_{t}\right)$ and the localizing conditions $g_{j} \cdot \Lambda \succeq 0$ (i.e. $\Lambda\left(g_{j} p^{2}\right) \geq 0$ for all $\left.p \in \mathbb{R}[\mathbf{x}]_{t-d_{j}}\right)$ aim to restrict the search for a representing measure supported by the set $K$ (cf. $[6,12])$. Using moment matrices, the program (3.1) can be formulated as an instance of semidefinite programming for which efficient algorithms exist (see e.g. $[21,22])$. We have: $p_{t}^{*} \leq p^{*}$, with equality if $H_{\Lambda}^{\mathcal{M}_{n, t}}$ is a flat extension of $H_{\Lambda}^{\mathcal{M}_{n, t-d}}$ for an optimum solution $\Lambda$ to $(3.1)\left(d:=\max _{j} d_{j}\right)$. In that case, the atoms of the representing measure (which exists by Corollary 1.3) are global minimizers of $p$ over the semi-algebraic set $K$ and they can be computed from $\Lambda$ [9]. Moreover, they are all the global minimizers when $H_{\Lambda}^{\mathcal{M}_{n, t}}$ has the maximum possible rank among all optimum solutions to the semidefinite program (3.1).

As shown in [13], the truncated moment problem also yields an algorithmic approach to the problem of computing the real roots to polynomial equations $g_{1}=0, \ldots, g_{m}=0$ (assuming their number is finite). Indeed, this amounts to finding all global minimizers to a constant polynomial, say $p=0$, over the real variety $K:=\left\{x \in \mathbb{R}^{n} \mid g_{j}(x)=0 \forall j=1, \ldots, m\right\}$. Consider the semidefinite program (3.1) where the localizing conditions now read $g_{j} \cdot \Lambda=0 \forall j$. For $t$ large enough, the program (3.1) has a maximum rank solution which is a flat extension and thus, as noted above, all points of $K$ can be computed from this solution. See [13] for details.

A concern in this type of approach is the size of the matrices appearing in the semidefinite program (3.1). In order to improve the practical applicability of this approach, it is crucial to derive semidefinite programs involving matrices of moderate sizes. For this one may want to consider moment matrices indexed by sparse sets of monomials instead of the full degree levels $\mathcal{M}_{n, t}$. This is where our new sparse flat extension theorem may become very useful. It will be used, in particular, in [11].

The approach in [13] also permits to find the real radical of the ideal generated by the polynomials $g_{1}, \ldots, g_{m}$. Indeed, if $\Lambda \in(\mathbb{R}[\mathbf{x}])^{*}$ is positive, then the kernel of its Hankel operator $H_{\Lambda}$ is a real radical ideal [15] and, 
under the conditions of Theorem 3.2, $\operatorname{ker} H_{\Lambda}^{\mathcal{C}^{+}}$generates a real radical ideal. These facts explain the relevance of moment matrices and Hankel operators to the problem of finding the real radical of a polynomial ideal. For instance, this permits to weaken the assumptions in Proposition 4.1 of [13] and to strengthen its conclusions; more precisely, we do not need to assume the commutativity of the operators $\chi_{i}$ 's (as this holds automatically, by Lemma 2.1) and we can claim that the returned ideal is real radical (by the above argument).

\section{References}

[1] N. I. Akhiezer, The Classical Moment Problem, Hafner, New York, 1965.

[2] C. Berg, J. P. R. Christensen and P. Ressel, Positive definite functions on Abelian semigroups, Math. Ann. 223 (1976), 253-272.

[3] C. Berg and P. H. Maserick, Exponentially bounded positive definite functions, Illinois J. Math. 28 (1984), 162-179.

[4] R. E. Curto And L. A. Fialkow, Solution of the truncated complex moment problem for flat data, Memoirs of the American Mathematical Society, 119, Amer. Math. Soc., Providence, RI, 1996.

[5] R. E. Curto And L. A. Fialkow, Flat extensions of positive moment matrices: recursively generated relations, Memoirs of the American Mathematical Society, 648, Amer. Math. Soc., Providence, RI, 1998.

[6] R. E. Curto and L. A. Fialkow, The truncated complex K-moment problem, Trans. Amer. Math. Soc. 352 (2000), 2825-2855.

[7] B. Fuglede, The multidimensional moment problem. Expositiones Mathematicae 1 (1983), 47-65.

[8] H. Hamburger, Über eine Erweiterung des Stieltjesschen Momentproblems, Parts I, II, III. Math. Ann. 81 (1920), 235-319, and 82 (1921), 20-164, 168-187.

[9] D. Henrion and J. B. Lasserre, Detecting global optimality and extracting solutions in GloptiPoly, In: Positive Polynomials in Control, D. Henrion and A. Garulli (eds.), Lecture Notes on Control and Information Sciences 312 (2005), 293-310, Springer, Berlin.

[10] A. Kehrein, M. Kreuzer, And L. Robbiano, An algebraist's view on border bases. Solving Polynomial Equations-Foundations, Algorithms and Applications, A. Dickenstein and I. Z. Emiris (eds.), pp. 169-202. Springer, Berlin, 2005 .

[11] J. B. LASserre ET AL., Moment matrices, border bases and real radical ideals, In preparation.

[12] J. B. Lasserre, Global optimization with polynomials and the problem of moments, SIAM Journal on Optimization 11 (2001), 796-817.

[13] J. B. Lasserre, M. Laurent, and P. Rostalski, Semidefinite characterization and computation of zero-dimensional real radical ideals, Found. Comput. Math. 8 (2008), 607-647. 
[14] J. B. Lasserre, M. Laurent, and P. Rostalski, A prolongation-projection algorithm for computing the finite real variety of an ideal, Theoret. Comput. Sci. 410 (2009), 2685-2700.

[15] M. Laurent, Revisiting two theorems of Curto and Fialkow on moment matrices, Proceedings of the American Mathematical Society 133 (2005), 2965-2976.

[16] M. Laurent, Sums of squares, moment matrices and optimization over polynomials, in: Emerging Applications of Algebraic Geometry, IMA Volumes in Mathematics and its Applications, 149, M. Putinar and S. Sullivant (eds.), Springer-Verlag, Berlin, pp. 157-270, 2009.

[17] M. Marshall, Positive Polynomials and Sums of Squares, Mathematical Surveys and Monographs, 146 AMS, Providence, 2008.

[18] B. Mourrain, A new criterion for normal form algorithms, In: Proceedings of the 13th International Symposium on Applied Algebra, Algebraic Algorithms and Error-Correcting Codes, H. Imai, S. Lin, and A. Poli (eds.) Lecture Notes In Computer Science, 1719 pp. 430-443. Springer-Verlag, Berlin, 1999.

[19] B. Mourrain And V. Y. Pan, Multivariate Polynomials, Duality, and Structured Matrices, J. Complexity 16 (2000), 110-180.

[20] J. Stochel, Solving the truncated moment problem solves the moment problem, Glasgow Journal of Mathematics 43 (2001), 335-341.

[21] L. Vandenberghe And S. Boyd, Semidefinite Programming, SIAM Review 38 (1996), 49-95.

[22] H. Wolkowicz, R. Saigal, and L. Vandeberghe (eds.), Handbook of Semidefinite Programming, Kluwer, Dordrecht, 2000.

\section{Monique Laurent}

Centrum Wiskunde \& Informatica (CWI),

Science Park I23,

1098 XG, Amsterdam,

The Netherlands

e-mail: M.Laurent@cwi.nl

BERnARd Mourrain

GALAAD, INRIA Méditerranée, BP 93, 06902 Sophia Antipolis,

France

e-mail: mourrain@sophia.inria.fr

Received: 14 December 2008 